\title{
A LOGARITHMIC MEAN AND INTERSECTIONS OF OSCULATING HYPERPLANES IN $R^{n}$
}

\section{ALAN HORWITZ}

Abstract. We discuss a special case of the means defined in [1]. Let $C$ be the curve in $R^{n}$ with vector equation $\hat{\alpha}(t)=\left\langle t, t \log t, \ldots, t(\log t)^{n-1}\right\rangle$. Let $0<a_{1}<\cdots<a_{n}$ and let $O_{k}$ be the osculating hyperplane to $C$ at $a_{k}$. Then we show that $O_{1}, \ldots, O_{n}$ have a unique point of intersection, $P=\left(i_{1}, \ldots, i_{n}\right) \in R^{n}$, and in particular, $i_{1}$ equals the mean

the logarithmic mean of Neuman.

$$
M\left(a_{1}, \ldots, a_{n}\right)=(n-1) ! \sum_{j=1}^{n} \frac{a_{j}}{\prod_{\substack{i=1 \\ i \neq j}}^{n}\left(\ln a_{j}-\ln a_{i}\right)},
$$

Mathematics subject classification (2010): 25E60, $26 \mathrm{~B} 99$.

Keywords and phrases: logarithmic mean; osculating hyperplane; Wronskian.

\section{REFERENCES}

[1] A. Horwitz, Means, Generalized Divided Differences, and Intersections of Osculating Hyperplanes, J. Math. Anal. Appl. 200 (1996), 126-148.

[2] Jorma K. Merikos Ki, Extending Means Of Two Variables To Several Variables, JIPAM, Volume 5, Issue 3, Article 65, 2004.

[3] S. Mustonen, Logarithmic mean for several arguments, (2002), [http://www.survo.fi/papers/logmean.pdf].

[4] Edward Neuman, The Weighted Logarithmic Mean, J. Math. Anal. Appl 188 (1994), 885-900.

[5] A. O. Pittenger, The logarithmic mean in $n$ variables, Amer. Math. Monthly 92 (1985), 99-104.

[6] K. Stolarsky, Generalizations of the logarithmic mean, Math. Mag. 48 (1975), 87-92.

[7] Zhen-Gang Xiao And Zhi-Hua Zhang, The Inequalities $G \leqslant L \leqslant I \leqslant A$ in $n$ Variables, JIPAM, Volume 4, Issue 2, Article 39, 2003. 